\title{
891 S-531011, A NOVEL ANTI-HUMAN CCR8 ANTIBODY: ANTIBODY SCREENING AND EVALUATION OF BIOLOGICAL PROFILES
}

${ }^{1}$ Mai Yoshikawa*, 'Yoji Nagira, 'Morio Nagira, 'Tetsuya Yoshida, 'Shinpei Yoshida, ${ }^{1}$ Tetsuyoshi Soh, ${ }^{2}$ Hisashi Wada, ${ }^{2}$ Naganari Ohkura, ${ }^{1}$ Tatsuya Takahashi. ${ }^{1}$ Shionogi and Co., Ltd., Osaka, Japan; ${ }^{2}$ Osaka University, Osaka, Japan

Background Regulatory $\mathrm{T}$ cells (Tregs) are involved in tumor progression and inhibition of anti-tumor immune responses by promotion of immunological tolerance in the tumor microenvironment. On the other hand, Treg cells in peripheral blood are also essential role in preventing autoimmunity and uncontrolled inflammation. So, selective control of tumor infiltrating Treg cells might be an attractive approach of immune-oncology therapies without disrupting their systemic anti-inflammatory functions. Here, we focused on CCR8 (C-C motif chemokine receptor 8) as a target molecule which was selectively and highly expressed on tumor-infiltrating Tregs and developed a novel anti-human CCR8 specific antibody.

Methods We immunized mice with human CCR8 by our original immunization method which could strongly induce antibodies for membrane proteins, and then constructed hybridoma cells. Anti-human CCR8 (human CCR4 as a negative control) binding assay and human CCL1-CCR8 neutralizing assay were simultaneously performed by using supernatants of hybridoma cells to isolate human CCR8 specific strong-neutralizing antibodies. After humanization and affinity maturation of some selected clones, we selected our lead antibody by binding specificity, neutralizing activity, antibody dependent cellular cytotoxicity (ADCC) and thermodynamic stability as index.

Results We rapidly induced human CCR8 specific antibodies in mouse with our unique immunization methods and constructed thousands of hybridomas secreting anti-human CCR8 antibodies. We also successfully humanized some of lead antibodies which show high affinity and specificity and isolated novel anti-human CCR8 specific humanized antibody S531011 as our development antibody after affinity maturation. S-531011 selectively recognizes human CCR8 on the surface of tumor-infiltrating Tregs and shows strong ADCC. While human CCL1 is known as a dominant ligand of CCR8 which binds extracellular loop2 and N-terminal of CCR8, S-531011 recognizes similar epitopes and effectively neutralizes CCL1CCR8 signaling. Furthermore, S-531011 also shows favorable blood kinetics in vivo and potently inhibits tumor growth in tumor bearing human CCR8 knock-in mouse model.

Conclusions We develop S-531011, a novel anti-human CCR8 humanized antibody which could selectively recognize and deplete tumor infiltrating Tregs. Based on our pre-clinical data, S-531011 has strong anti-tumor effect and we expect that it might be a potent novel tumor immuno-therapeutic agent with fewer side effect.

Ethics Approval The present study was approved by the Institutional Ethics Committee of Osaka University Hospital (approved number: 13266-15). Animal studies were approved by the Institutional Animal Care and Use Committee (approved number: S20192D, S20197D and S20198D).

http://dx.doi.org/10.1136/jitc-2021-SITC2021.891 